\title{
Microscopic black hole and uncertainty principle with minimal length and momentum
}

\author{
M. M. Stetsko* \\ Department of Theoretical Physics, Ivan Franko National University of Lviv, \\ 12 Drahomanov Str., Lviv, UA-79005, Ukraine
}

December 12, 2018

\begin{abstract}
We investigate a microscopic black hole in case of modified generalized uncertainty principle with a minimal uncertainty in position as well as in momentum. We calculate thermodynamical functions of a Schwarzschild black hole such as temperature, entropy and heat capacity. It is shown that incorporation of minimal uncertainty in momentum leads to minimal temperature of a black hole. Minimal temperature gives rise to appearance of a phase transition. Emission rate equation and black hole's evaporation time are also obtained.
\end{abstract}

Keywords: generalized uncertainty principle, black hole, thermodynamics

PACS numbers: 04.60.Bc, 04.70.Dy

\section{Introduction}

Quantum black holes have been intensively investigated for recent years. This interest was mainly stimulated by the development of high energy physics. One of the most promising open issues of high energy physics is the development of theories with extra space-like dimensions. It was proposed that extra space-like dimensions can lower the Planck scale to the TeV region 1, 2, 3, 4,. It is expected that particle collisions with center-of-mass energy above the Planck scale and impact parameter smaller than the horizon radius can be a source for producing micro black holes and branes [5, 6, 7, 8, 9, 10, 11. (Some critical remarks to this scenario can be found in [12, 13]). So the energy sufficient for producing of micro black holes can in principle be achievable even for present generation of particle accelerators. The lowering of the Planck scale possibly allows to by-pass the hierarchy problem. Identification of the ultraviolet cutoff scale with electroweak energy one $\Lambda_{E W}$ can lead to radiative stability without using additional assumptions such as supersymmetry or technicolor. It is considered that the unification of gravity with other gauge interactions at electroweak scales can be obtained in theories with extra dimensions. Another important feature of theories with extra dimensions is violation of Newton's law of gravitation $\sim 1 / r^{2}$ and this deviation can be possibly probed in the nearest feature.

*E-mail: mstetsko@gmail.com, mykola@ktf.franko.lviv.ua 
Evolution of micro black holes, formed in high energy collisions can be divided into several stages, so called phases: loss of gauge charges and multipole momenta (balding phase), loss of angular momentum (spin-down phase) and the last one is so called Schwarzschild phase when a black hole loses energy via Hawking radiation. The final stage of Schwarzschild phase is not well understood due to the lack of fully-fledged theory of quantum gravity. Several attempts to understand this stage were made with the help of Generalized Uncertainty Principle (GUP) [14, 15, 16]. It was shown that GUP prevents complete evaporation of black holes leaving some remnants. Noncommutative geometry was also applied to quantum black holes. The key point in this approach is the noncommutativity inspired modification of black hole's metrics [17].

Another line of research that has renewed interest to micro black holes, especially with GUP, is cosmology. Recent investigations of the CMB power spectrum have brought an idea about preinflationary epoch [18, 19]. The preinflationary scenario proposed in [20] was based on the micro black hole production and a set of first principles such as generalized uncertainty principle and holographic principle. These principles allow one to obtain self-consistent description of the suppression of the CMB quadrupole without additional assumptions. As it was pointed out that GUP can lead to the matter domination in the preinflatory epoch. In spite of some success of matter dominant preinflation this scenario has some drawbacks and needs further investigation.

This paper is organized as follows. In the second section we consider modified generalized uncertainty principle with minimal position and momentum and obtain an expression for the black hole's temperature. In the third section we calculate entropy and heat capacity of black hole. In the fourth section emission rate relation is considered and black hole's evaporation time is calculated. The fifth section contains conclusions and some mathematical details are considered in Appendix.

\section{Uncertainty principle with minimal length and momentum and black hole's temperature}

Recent studies in string theory brought an idea about generalization of Heisenberg uncertainty principle 21, 22]. Similar suggestion was proposed to reconcile principles of Quantum Mechanics and General Relativity [23, 24]. Generalized Uncertainty Principle (GUP) takes form:

$$
\Delta p \Delta x \geqslant \frac{\hbar}{2}\left(1+\beta \Delta p^{2}\right)
$$

As it is known such a generalization leads to appearance of minimal uncertainty in position, so called minimal length. It was shown that gravity-induced decoherence gives rise to similar modification of uncertainty principle but with minimal uncertainty for momentum [25, 26, 27]. These two assumptions about minimal uncertainties in position and momentum might be merged in order to obtain modified generalized uncertainty with both minimal length and momentum. We can write uncertainty relation:

$$
\Delta x \Delta p \geqslant \frac{\hbar}{2}\left(1+\alpha \Delta x^{2}+\beta \Delta p^{2}\right)
$$

It is easy to show that $\Delta x \geqslant \Delta x_{\text {min }}=\hbar \sqrt{\beta} /\left(\sqrt{1-\hbar^{2} \alpha \beta}\right)$ and $\Delta p \geqslant \Delta p_{\text {min }}=\hbar \sqrt{\alpha} /\left(\sqrt{1-\hbar^{2} \alpha \beta}\right)$. We note that in order to get these minimal uncertainties parameters $\alpha, \beta$ must be positive and $\hbar^{2} \alpha \beta<1$. We also remark that uncertainty principle (2) was introduced after consideration of a gedanken experiment for the simultaneous measurement of position and momentum of a particle in de Sitter spacetime [28]. 
The uncertainty relation (2) can be obtained if we suppose that operators of position and momentum obey deformed commutation relation:

$$
[x, p]=i \hbar\left(1+\alpha x^{2}+\beta p^{2}\right),
$$

where parameters of deformation $\alpha$ and $\beta$ are assumed to be positive $(\alpha, \beta>0)$ and $\hbar^{2} \alpha \beta<1$. We note that commutation relation (3) and uncertainty principle (2) firstly appeared in quantum group investigations [29].

We point out that some troubles appear when one tries to generalize the algebra (3) for a case of higher dimension. Probably the main difficulty is to satisfy closedness condition. It was proposed to generalize commutation relation (3) in the following way [30, 31]:

$$
\left[x_{i}, p_{j}\right]=i \hbar\left(\delta_{i j}+\alpha x_{i} x_{j}+\beta p_{j} p_{i}+\sqrt{\alpha \beta}\left(p_{i} x_{j}+x_{j} p_{i}\right)\right) .
$$

Whereas other two commutation relations need to be written in a proper way to fulfil the Jacobi identity. It was also proposed the representation of operators obeying (44). We also point out that classical commutation relations that use classical counterpart of (4) are well defined (see Appendix).

Let us come back to the uncertainty (2) and rewrite it in the form:

$$
\frac{1}{\hbar \beta}\left(\Delta x-\sqrt{\Delta x^{2}\left(1-\hbar^{2} \alpha \beta\right)-\hbar^{2} \beta}\right) \leqslant \Delta p \leqslant \frac{1}{\hbar \beta}\left(\Delta x+\sqrt{\Delta x^{2}\left(1-\hbar^{2} \alpha \beta\right)-\hbar^{2} \beta}\right)
$$

It is easy to see that right hand side inequality does not recover ordinary uncertainty principle in the limit $\beta \rightarrow 0$ or (and) $\alpha \rightarrow 0$. So we obtain

$$
\Delta p \geqslant \frac{1}{\hbar \beta}\left(\Delta x-\sqrt{\Delta x^{2}\left(1-\hbar^{2} \alpha \beta\right)-\hbar^{2} \beta}\right)
$$

It was proposed that applying uncertainty principle to a black hole one can get Hawking temperature up to a some "calibration" factor [14. As it was pointed out by Adler and collaborators GUP prevents a black hole from complete evaporation and temperature catastrophe. That idea attracted a lot of attention and was generalized for the case of higher dimension [15, 16, 32].

Since evaporated particles appear just outside of the horizon surface it means that uncertainty in position for them can not be less than linear size of a black hole. If we assume that the uncertainty in position for a particle is less than linear size of the black hole it means that particle emerges inside the black hole and can not penetrate through the horizon surface. On the other hand if we suppose that the uncertainty in position is greater than linear size of the black hole then the emitted particle appears not on the outside of horizon surface but somewhere beyond it and as a consequence the temperature of the black hole will be different than it should be. To obtain temperature of the black hole we equate uncertainty in position for emitted particle to the double of Schwarzschild radius.

$$
\Delta x=2 R_{S} .
$$

Having used (6) and supposing that $\Delta E=c \Delta p$ we obtain the uncertainty in the energy of emitted particle.

$$
\Delta E=c \Delta p \geqslant \frac{c}{\hbar \beta}\left(2 R_{S}-\sqrt{4 R_{S}^{2}\left(1-\hbar^{2} \alpha \beta\right)-\hbar^{2} \beta}\right)
$$

Let us suppose that we deal with ordinary Hawking effect and consider Hawking radiation just outside the event horizon of a Schwarzschild black hole. We identify the 
uncertainty of energy $\Delta E$ given by the relation (8) with the thermal energy of an emitted particle. So we can use well-known energy-temperature relation $\Delta E=3 T$ (We have used thermal energy for photons and here we set Boltzmann constant equal to unity $k_{B}=1$ ). Taking into account all these remarks and substituting the mass $M$ of a black hole instead of its gravitational radius $R_{S}\left(R_{S}=2 G M / c^{2}\right)$ we obtain relation for the temperature of emitted particles

$$
T=\frac{c}{\pi \hbar \beta}\left(\frac{4 G M}{c^{2}}-\sqrt{\frac{16 G^{2} M^{2}}{c^{4}}\left(1-\hbar^{2} \alpha \beta\right)-\hbar^{2} \beta}\right)
$$

We remark that in the latter relation we have used "calibration" factor $\pi$ instead of 3 in order to get correct relation for Hawking temperature when parameters of deformation tend to zero $(\alpha, \beta \rightarrow 0)$. Probably the correct result for the Hawking temperature can be reproduced if one uses proper equipartition law on the horizon surface or just outside it. It was shown that number of degrees of freedom on the horizon surface is defined by its surface area [33. So it is worth expecting that proper equipartition law leads to correct "calibration" factor. It should be noted that Hawking temperature for the Schwarzschild-AdS black hole with modified GUP (2) was obtained in [34]. But in order to reproduce correct relation for the temperature of the black hole one of the deformation parameters was held fixed [34. Unfixing this parameter and taking Schwarzschild metrics instead of Schwarzschild-AdS one we arrive at the relation (9). We point out that uncertainty relation (2) can be used with Schwarzschild metrics because it can be derived without any assumption about the de Sitter spacetime. We also remark that temperature of Schwarzschild-AdS black hole with generalized uncertainty principle that leads to appearance of minimal momentum was firstly considered in [35].

It was pointed out [35] that uncertainty principle does not describe the origin of such effects as semiclassical wave scattering or particle tunnelling but only their consequence on the measurement process. To explain the origin of black hole's evaporation it is necessary to know the quantum states of the black hole from which the exact uncertainty principle follows. It was suggested that black holes thermodynamics is a low-energy effect of smallscale physics. It is known that any theory of quantum gravity contains some kind of uncertainty principle that reduces to Heisenberg principle at low energies. So black hole thermodynamics should not depend too much on the details of underlying quantum gravity theory [35]. This is in agreement with Visser's conclusion that Hawking radiation requires ordinary quantum mechanics and slowly evolving future horizon, so to explain black hole thermodynamics quantum gravity principles are not necessary [36].

Uncertainty relation (2) gives rise to the existence of a minimal mass of a black hole:

$$
M_{\min }=\frac{\hbar c^{2} \sqrt{\beta}}{4 G \sqrt{1-\hbar^{2} \alpha \beta}}=\frac{c M_{P l}^{2} \sqrt{\beta}}{4 \sqrt{1-\hbar^{2} \alpha \beta}},
$$

where $M_{P l}=\sqrt{\hbar c / G}$ is the Planck's mass. So according to the relation (9) it leads us to the finite temperature at the final point of Hawking radiation, which takes following form:

$$
T_{\text {final }}=\frac{c}{\pi \sqrt{\beta} \sqrt{1-\hbar^{2} \alpha \beta}}
$$

One can see that uncertainty relation (2) causes increasing of black hole's temperature in comparison with relation (1).

Minimal uncertainty in position given by relation (2) similarly as in case of (11) prevents us from a temperature catastrophe. It was proposed that generalized uncertainty principle (11) prevents black holes from complete evaporation in the same way as the standard 


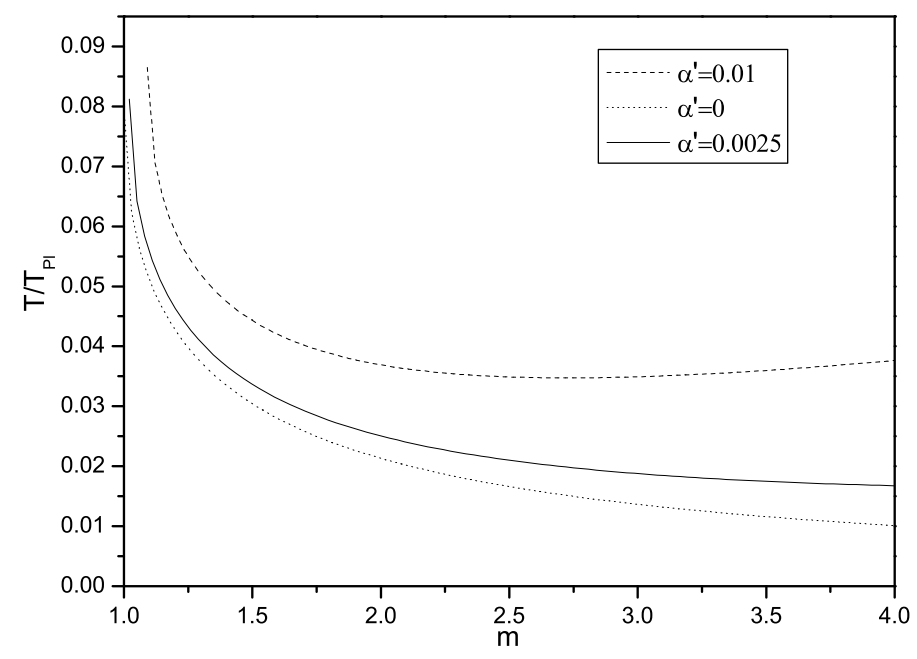

Figure 1: Temperature of a black hole as a function of mass. Temperature and mass are represented in Planck's units. So $m=M / M_{P l}$ denotes relation of black hole's mass to the Planck's mass. Deformation parameters are also written in dimensionless form: $\alpha^{\prime}=\alpha L_{P l}^{2}$ and $\beta^{\prime}=\hbar^{2} \beta / L_{P l}^{2}$. In order to get that the mass of a black hole at the final point of Hawking radiation to be equal to the Planck's mass (or $m=1$ ) when the parameter $\alpha^{\prime}=0$ we set $\beta^{\prime}=16$.

uncertainty principle prevents the hydrogen atom from collapse [15]. To make the influence of a minimal uncertainty in momentum clear let us investigate temperature as a function of $M$. Taking the derivative $\partial T / \partial M$ and equating it to zero one can find that temperature has an extremum point when the mass of a black hole reaches:

$$
M_{e x t}=\frac{c^{2}}{4 G \sqrt{\alpha\left(1-\hbar^{2} \alpha \beta\right)}} .
$$

It is easy to see that at this point temperature of the black hole takes minimal value:

$$
T_{\min }=\frac{c \hbar \sqrt{\alpha}}{\pi \sqrt{1-\hbar^{2} \alpha \beta}}
$$

When the black hole's mass is above $M_{\text {ext }}$, temperature (9) increases with the mass rise. When the mass is below $M_{\text {ext }}$ its decreasing leads to increasing of temperature. Similar mass-temperature dependence was obtained in case of Schwarzschild-AdS black hole with modified generalized principle (2). We note that minimal temperature is the consequence of a minimal uncertainty in momentum and it can appear for different black hole's metrics. Temperature as the function of mass is shown in Fig:1.

In the case of higher dimensional space one should use Schwarzschild-Tangherlini metrics [37, 38]. The horizon radius of a black hole takes form:

$$
R_{S}=\left(\frac{16 \pi G_{d} M}{(d-2) \Omega_{d-2} c^{2}}\right)^{1 /(d-3)}
$$

Where $d$ is the dimension of space-time, $G_{d}$ is the $d$-dimensional gravitational constant and $\Omega_{d-2}$ is the surface area of $d-2$-dimensional unit hypersphere. So the temperature 
of a $d$-dimensional black hole takes form:

$$
T=\frac{(d-3) c}{\pi \hbar \beta}\left[2\left(\frac{16 \pi G_{d} M}{(d-2) \Omega_{d-2} c^{2}}\right)^{1 /(d-3)}-\sqrt{4\left(\frac{16 \pi G_{d} M}{(d-2) \Omega_{d-2} c^{2}}\right)^{2 /(d-3)}\left(1-\hbar^{2} \alpha \beta\right)-\hbar^{2} \beta}\right]
$$

As it was pointed out in [16] generalized uncertainty relation (10) for the black hole in the case of higher dimensions should be replaced by another one due to the fact that distances can not be probed below the Schwarzschild radius (14). So according to [16] instead of generalized uncertainty principle (11) in $d=4+n$-dimensional space-time one should write:

$$
\Delta p \Delta x \geqslant \frac{\hbar}{2}\left(1+\gamma \Delta p^{\frac{n+2}{n+1}}\right),
$$

where $n$ is the number of additional spacelike dimensions. It was noted [16] that at high energies stringy GUP (11) can give an uncertainty $\Delta x$ larger than the size of a black hole itself. But scenarios with extra dimensions suggest that all gauge interactions apart from gravity live on $3+1$ dimensional brane [1, 2, 3, 4. So modification of GUP (16) is valid only for gravitons, whereas for photons and other SM particles emitted by a black hole it is necessary to use uncertainty principle (11) or (and) (2). Taking into account all the above remarks we can interpret the relation (15) as some upper bound for temperature of emitted gravitons.

\section{Entropy and heat capacity of a black hole}

Having calculated black hole's temperature we can find black hole's entropy using wellknown thermodynamical relation:

$$
d S=\frac{c^{2}}{T} d M
$$

After integration we obtain:

$$
\begin{array}{r}
S=\frac{\pi c^{3}}{4 \hbar G \alpha}\left(\ln \left|\frac{4 G M-\sqrt{16 G^{2} M^{2}\left(1-\hbar^{2} \alpha \beta\right)-\hbar^{2} c^{4} \beta}}{\hbar c^{2} \sqrt{\beta}}\right|+\right. \\
\left.\sqrt{1-\hbar^{2} \alpha \beta} \ln \left|\frac{\sqrt{16 G^{2} M^{2}\left(1-\hbar^{2} \alpha \beta\right)-\hbar^{2} c^{4} \beta}+4 G M \sqrt{1-\hbar^{2} \alpha \beta}}{\hbar c^{2} \sqrt{\beta}}\right|\right)
\end{array}
$$

The last formula reproduces well-known relation for the Bekenstein-Hawking entropy in the limit $\alpha, \beta \rightarrow 0$. We can suppose that parameter $\alpha$ is small in comparing with $\beta$ so we can expand the right hand side of equation (18) into the series over a small parameter $\alpha$.

$$
\begin{array}{r}
S=\frac{\pi}{4 \hbar c G}\left(8 G^{2} M^{2}+2 G M\right. \\
\sqrt{16 G^{2} M^{2}-\hbar^{2} c^{4} \beta}-\frac{\hbar^{2} c^{4} \beta}{2} \ln \left(\frac{4 G M+\sqrt{16 G^{2} M^{2}-\hbar^{2} c^{4} \beta}}{\hbar c^{2} \sqrt{\beta}}\right) \\
-\frac{\alpha}{c^{4}}\left[64 G^{4} M^{4}+G M \sqrt{16 G^{2} M^{2}-\hbar^{2} c^{4} \beta}\left(\frac{\hbar^{2} c^{4} \beta}{2}+16 G^{2} M^{2}\right)\right. \\
\left.\left.+\frac{\hbar^{4} c^{4} \beta^{2}}{2} \ln \left(\frac{4 G M+\sqrt{16 G^{2} M^{2}-\hbar^{2} c^{4} \beta}}{\hbar c^{2} \sqrt{\beta}}\right)\right]\right)
\end{array}
$$

In the limit $\alpha \rightarrow 0$ we reproduce the expression for the entropy in the presence of a minimal length [14]. Expression (19) shows that including $\alpha$-dependent terms lead to decreasing of entropy similarly as we have in the case with $\beta$-terms only. 


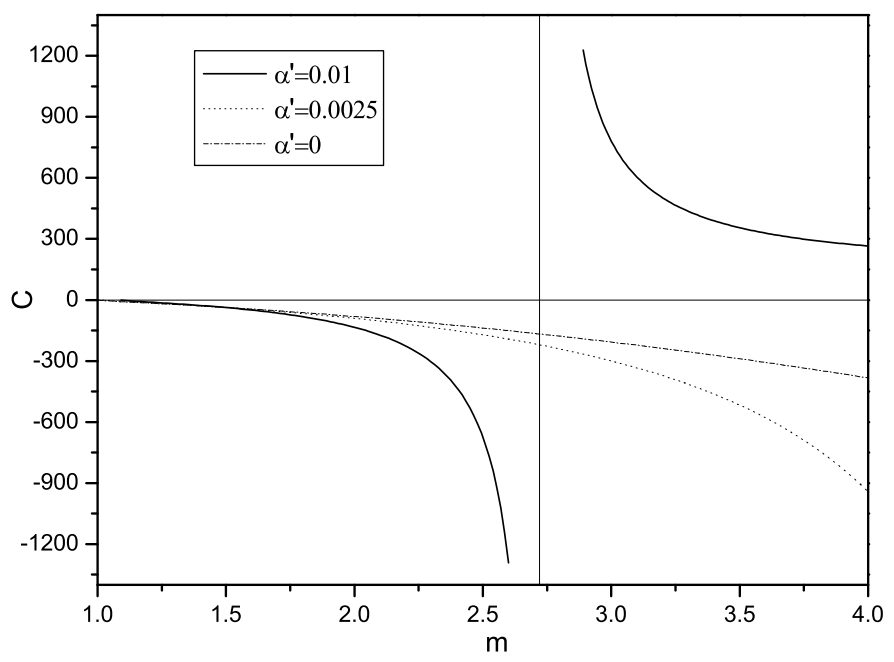

Figure 2: Heat capacity as a function of mass.

To calculate the heat capacity of a black hole we use a well-known thermodynamical relation:

$$
C=T \frac{\partial S}{\partial T}=\frac{\partial E}{\partial T}
$$

In our case temperature and entropy are represented as functions of black hole's mass $M$ (or diameter $R$ ). So we write the heat capacity as a function of mass $M$.

$$
C=\frac{\pi \hbar c^{3} \beta}{4 G} \frac{\sqrt{16 G^{2} M^{2}\left(1-\hbar^{2} \alpha \beta\right)-\hbar^{2} c^{4} \beta}}{\sqrt{16 G^{2} M^{2}\left(1-\hbar^{2} \alpha \beta\right)-\hbar^{2} c^{4} \beta}-4 G M\left(1-\hbar^{2} \alpha \beta\right)}
$$

Let us investigate the latter relation in details. It is easy to make yourself sure that when mass is below $M_{e x t}$ heat capacity is negative and it is equal to zero when the mass reaches $M_{\min }$. When the mass of black hole is above $M_{\text {ext }}$ heat capacity is positive and tends to a finite value when mass goes to infinity. So $M_{e x t}$ is the discontinuity point for the heat capacity. Heat capacity as a function of mass is represented in Fig,2, Negative heat capacity shows that thermodynamical system is unstable and tends to decay. Whereas positive heat capacity makes system stable. So at the extremum point we have phase transition. One can conclude that the phase transition is caused by the modified GUP (2) but not particular choice of black hole's metrics. As it has already been noted modified GUP (21) related to commutation relation (4) but it is known that the choice of commutation relations that give the same uncertainty principle is non unique. So modified GUP (2) might be obtained for more general case of commutation relations. We point out that behaviour of heat capacity near the phase transition point is similar to behavior of heat capacity in case of the well-known Hawking-Page phase transition [39, 40]. Phase transition also appears when the modifications of black hole's metrics caused by nonlocal effects or noncommutative geometry are taken into account [41]. But in that case stable and unstable phases are exchanged their places in comparison with our result. We also note that extremum point $M_{\text {ext }}$ is an inflection point for entropy. Entropy as a function of mass is shown in Fig 3 , 


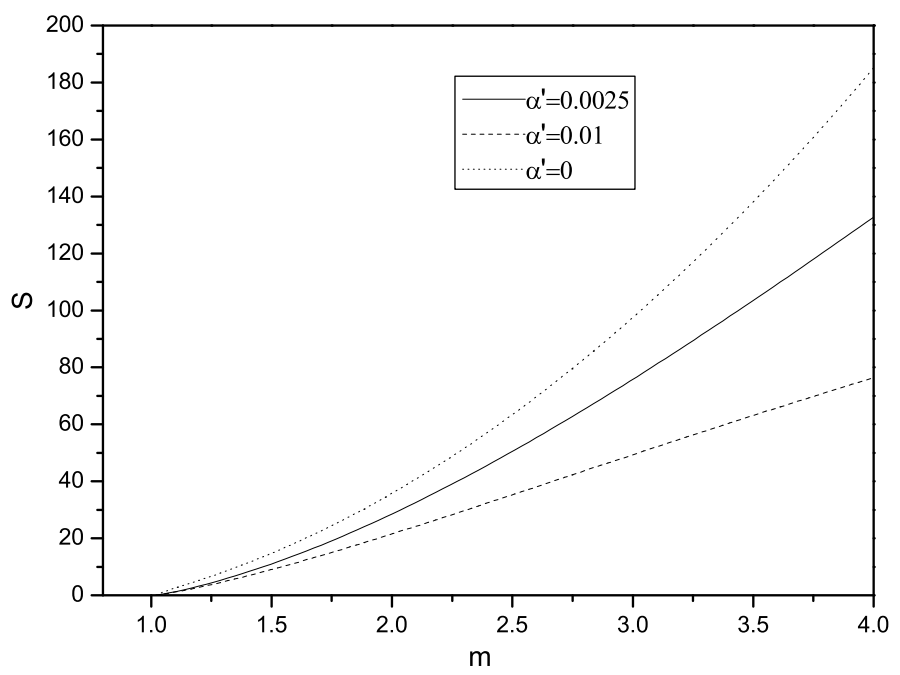

Figure 3: Entropy as a function of mass

\section{Emission rate equation and evaporation time of a black hole}

In this section evaporation of a micro black hole is studied. Similar problem with GUP was firstly considered in work [14]. This topic was investigated in details in [15, 16]. To examine quantum black hole's evaporation different approaches are used. Probably the simplest one is to make use of Stefan-Boltzmann law for the black-body radiation which is based on an assumption that we have a thermodynamical system with fixed temperature so we consider canonical ensemble. We note that the Stefan-Boltzmann law gets new correction caused by deformation of commutation relations [15, 16, 42. As it was pointed out in [43, 44] canonical description for a micro black hole is adequate when the energy of emitted particles (e.g. photons, gravitons) is small in comparison with the energy of a black hole itself. If the energy of emitted particles is comparable with the energy of a black hole itself one should use microcanonical description. When the energy of black hole is large in comparison with the energy of emitted particles both of them give the same result. Here we use canonical description, microcanonical one will be considered elsewhere. We also point out that statistical mechanics in the case of the deformed commutation relations was considered in [45].

Let us examine equilibrium radiation caused by a black hole. We suppose that that black hole is placed inside a sphere of given radius $R$ and system is considered under a fixed temperature $T$. For generality we consider $D$-dimensional case $(D=3+n)$. So the energy of evaporated particles can be calculated as follows (photons, gravitons):

$$
E=\frac{g}{(2 \pi \hbar)^{D}} \int \frac{d^{D} x d^{D} p}{1+\alpha x^{2}+\beta p^{2}+2 \sqrt{\alpha \beta}(\mathbf{x}, \mathbf{p})} \frac{\varepsilon(p) \Gamma(p)}{e^{\varepsilon(p) / T}-1}
$$

where $\varepsilon(p)$ is the dispersion relation for emitted particles, $\Gamma(p)$ is the greybody factor and $g$ is the degeneracy factor. In the case of photons we put $\varepsilon(p)=c p$ and $g=2$. The same dispersion relation can be considered for gravitons but the degeneracy factor is different. We also suppose that greybody factor is equal to unity $\Gamma(p)=1$ (blackbody radiation). Let us remind that emission of gravitons takes place in $d=4+n$ - dimensional bulk spacetime whereas photons and other gauge particles are emitted on 3+1-dimensional brane. 
We note that suppositions we have made do not allow to calculate integral exactly (22) even if one of the deformation parameters is absent. To calculate it we assume that parameters $\alpha$ and $\beta$ are small and develop weight function under integral into the series over deformation parameters. So the latter relation can be rewritten in the form:

$$
E=\frac{g}{(2 \pi \hbar)^{D}} \int d^{D} x d^{D} p\left(1-\alpha x^{2}-\beta p^{2}-2 \sqrt{\alpha \beta}(\mathbf{x}, \mathbf{p})\right) \frac{c p}{e^{c p / T}-1}
$$

After integration we obtain:

$$
\begin{array}{r}
E=\frac{2 g}{(2 \sqrt{\pi} c \hbar)^{D}} \frac{D ! \zeta(D+1)}{\Gamma(D / 2)}\left[\left(V-\frac{D^{1+2 / D}}{D+2}\left(\frac{\Gamma(D / 2)}{2 \pi^{D / 2}}\right)^{2 / D} \alpha V^{1+2 / D}\right) T^{D+1}\right. \\
\left.-\frac{(D+2) ! \zeta(D+3)}{D ! \zeta(D+1)} \beta V T^{D+3}\right]
\end{array}
$$

where $V=2 \pi^{D / 2} R^{D} /(D \Gamma(D / 2))$ is the volume of $D$-dimensional sphere. It should be noted that the energy of radiation nonlinearly depends on the volume. In three dimensional case we find:

$$
E=\frac{g}{(2 \pi \hbar)^{3}}\left[\left(V-\frac{3}{5}\left(\frac{3}{4 \pi}\right)^{2 / 3} \alpha V^{5 / 3}\right) \frac{4 \pi^{5}}{15 c^{3}} T^{4}-\frac{32 \pi^{7}}{63 c^{3}} \beta V T^{6}\right]
$$

To obtain emission rate equation we suppose that particles are emitted by sphere with radius $R_{S}$. So the total energy $d E$ emitted during the period of time $d t$ can be written in the form:

$\frac{d E}{d t}=-\frac{4 g c}{(2 \hbar c)^{D}} \frac{D ! \zeta(D+1)}{(\Gamma(D / 2))^{2}}\left[\left(R_{S}^{D-1}-\alpha R_{S}^{D+1}\right) T^{D+1}-\frac{(D+2) ! \zeta(D+3)}{D ! \zeta(D+1)} \beta R_{S}^{D-1} \frac{T^{D+3}}{c^{2}}\right]$

Where $R_{S}$ is the Schwarzschild radius. For photons in three dimensional case we obtain:

$$
\frac{d E}{d t}=-\frac{4 \pi^{3}}{15 c^{2} \hbar^{3}}\left(\left(R_{S}^{2}-\alpha R_{S}^{4}\right) T^{4}-\frac{40}{21} \frac{\pi^{2} \beta}{c^{2}} R_{S}^{2} T^{6}\right)
$$

In the limit when parameters of deformation tens to zero the last equation gives ordinary Stefan-Boltzmann law for surface of a black hole. At the final point of Hawking radiation, when the temperature and Schwarzschild radius reach $T_{\text {final }}$ and $R_{\text {min }}$ respectively, the emission rate is finite:

$$
\left.\frac{d E}{d t}\right|_{\text {final }}=\frac{c^{2}}{60 \hbar \beta\left(1-\hbar^{2} \alpha \beta\right)^{4}}\left(\frac{76}{21}+5 \hbar^{2} \alpha \beta\right) .
$$

Similarly as in the case of GUP [15] at the final point of Hawking radiation emission rate is finite. It was supposed that when the final stage has been reached, the black hole evaporates completely by emitting a hard Planck-size quantum with maximum temperature in a finite period of time proportional to the Planck's time [15]. At the same time heat capacity (21) tends to zero when the mass reaches $M_{\text {min }}$. It means that black hole cannot exchange heat with surroundings at the final point of Hawking radiation.

Now using relation (9) and representing Schwarzschild radius as a function of a black hole's mass we can write equation for the emission rate of a black hole. Since we have calculated the energy of emitted radiation up to the first order over deformation parameters we estimate emission rate up to first order too. So equation (27) can be written in the form:

$$
\frac{d M}{d t}=-\frac{\hbar c^{4}}{3840 \pi G^{2} M^{2}}\left(1+60 \alpha \frac{G^{2} M^{2}}{c^{4}}+\frac{11}{336} \beta \frac{\hbar^{2} c^{4}}{G^{2} M^{2}}\right)
$$




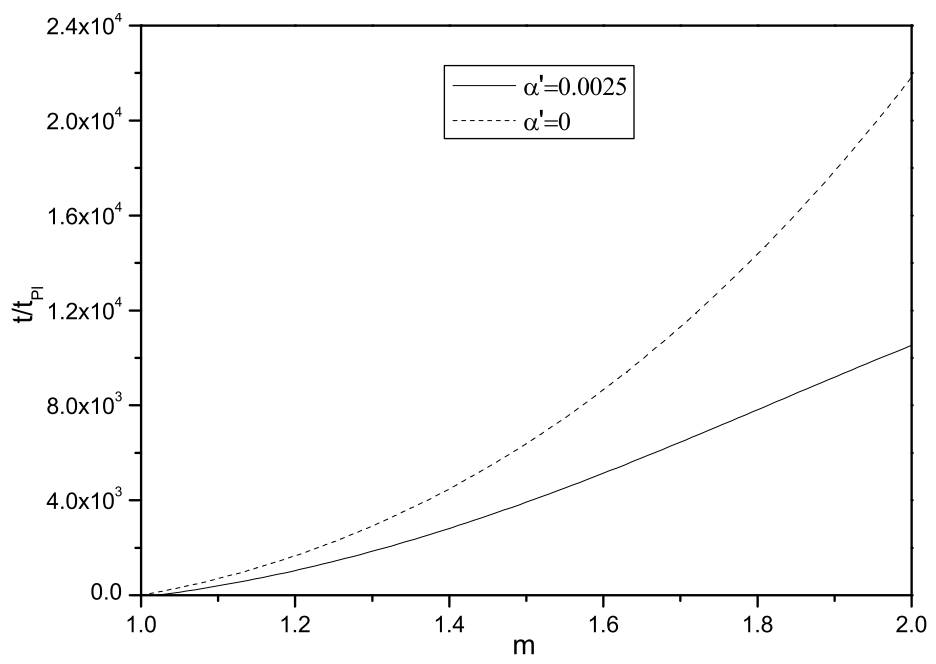

Figure 4: Evaporation time as a function of mass

Having integrated the last equation we obtain simple expression for evaporation time of a black hole:

$$
\frac{1}{3}\left(M_{\text {min }}^{3}-M^{3}\right)-12 \frac{\alpha G^{2}}{c^{4}}\left(M_{\text {min }}^{5}-M^{5}\right)-\frac{11}{336} \frac{\hbar^{2} \beta c^{4}}{G^{2}}\left(M_{\text {min }}-M\right)=-\frac{\hbar c^{4}}{3840 \pi G^{2}} t
$$

One can see that modification of generalized uncertainty principle (2) leads to further decrease in black hole's evaporation time in comparison with GUP (11). Evaporation time as a function of mass is shown in Fig, 4

\section{Conclusions}

We have considered microscopic black hole with modified GUP (2) leading to the appearance of a minimal length as well as minimal momentum. Uncertainty relation allows one to obtain thermodynamical functions of a black hole in a very simple way. Therefore it was the main motivation to investigate the black hole's thermodynamics under a bit more general assumption such as generalized uncertainty principle [14, 15]. Modification of GUP (2) gives rise to some new features in black hole's thermodynamics. Similarly as in case of GUP (11) black hole has finite final temperature which is caused by a minimal uncertainty in position. However, in contrast to GUP (1) modified GUP (2) leads also to minimal temperature (13). Similar result was firstly obtained in [34 but there Schwarzschild-AdS black hole's metrics was used and a specific choice for one of the deformation parameters was made. This minimal temperature causes important influence on black hole's thermodynamics. The point of minimal temperature is a point of discontinuity for heat capacity. As we have already pointed out when the mass of a black hole is below $M_{\text {ext }}(12)$ heat capacity is negative and it tells us about thermodynamical instability. So in this case black hole tends to decay. When the black hole mass is above $M_{\text {ext }}$ heat capacity is positive and black hole is thermodynamically stable. We also note that although modified GUP (2) is related to Snyder-de Sitter commutation relations it can obtained for a more general kind of commutation relations. We can conclude that phase transition which appears due to the presence of minimal momentum and it is possible for different black holes metrics.

We also investigated thermal radiation of a Schwarzschild black hole. We obtained the emission rate equation which is based on modified Stefan-Boltzmann law. Then we used 
it to calculate the evaporation time of a Schwarzschild black hole. In comparison with ordinary GUP (11) modified GUP (2) makes evaporation time of a black hole shorter. We also point out that calculations of evaporation time are valid only for unstable phase of a black hole when its mass is below $M_{\text {ext }}$.

\section{Appendix}

In order to obtain correct relation for the black body radiation spectrum we should modify Liouville theorem of the classical mechanics caused by a deformation of commutation relations. So we have to find an element of phase-space volume that is invariant under time evolution. To do this let us write classical equations of motion supposing that position and momentum coordinates are obeyed the classical variant of the algebra (4). In order to obtain the classical commutation relations the standard procedure is used:

$$
\frac{1}{i \hbar}[\hat{A}, \hat{B}] \Rightarrow\{A, B\}
$$

Under this assumption deformed commutation relation (44) takes following form:

$$
\left\{x_{i}, p_{j}\right\}=\delta_{i j}+\alpha x_{i} x_{j}+\beta p_{i} p_{j}+2 \sqrt{\alpha \beta} x_{j} p_{i} .
$$

As it has already been pointed out in the classical case commutation relation (32) forms a well defined algebra. Other two commutation relations takes form [30, 31]:

$$
\left\{x_{i}, x_{j}\right\}=\beta J_{i j} ; \quad\left\{p_{i}, p_{j}\right\}=\alpha J_{i j},
$$

where $J_{i j}$ are the components of angular momentum.

Hamilton's equations for the time derivatives of position and momentum read:

$$
\begin{gathered}
\dot{x}_{i}=\left\{x_{i}, H\right\}=\left\{x_{i}, x_{j}\right\} \frac{\partial H}{\partial x_{j}}+\left\{x_{i}, p_{j}\right\} \frac{\partial H}{\partial p_{j}}, \\
\dot{p}_{i}=\left\{p_{i}, H\right\}=\left\{p_{i}, x_{j}\right\} \frac{\partial H}{\partial x_{j}}+\left\{p_{i}, p_{j}\right\} \frac{\partial H}{\partial p_{j}} .
\end{gathered}
$$

Considering evolution of a system during an infinitesimal period of time we obtain:

$$
\begin{array}{r}
x_{i}^{\prime}=x_{i}+\delta x_{i} \\
p_{i}^{\prime}=p_{i}+\delta p_{i} .
\end{array}
$$

Here

$$
\begin{gathered}
\delta x_{i}=\dot{x}_{i} \delta t=\left(\left\{x_{i}, x_{j}\right\} \frac{\partial H}{\partial x_{j}}+\left\{x_{i}, p_{j}\right\} \frac{\partial H}{\partial p_{j}}\right) \delta t \\
\delta p_{i}=\dot{p}_{i} \delta t=\left(\left\{p_{i}, x_{j}\right\} \frac{\partial H}{\partial x_{j}}+\left\{p_{i}, p_{j}\right\} \frac{\partial H}{\partial p_{j}}\right) \delta t .
\end{gathered}
$$

After infinitesimal evolution an element of phase-space volume changes:

$$
d^{D} x^{\prime} d^{D} p^{\prime}=\left|\frac{\partial\left(x_{1}^{\prime}, \ldots, x_{D}^{\prime}, p_{1}^{\prime}, \ldots, p_{D}^{\prime}\right)}{\partial\left(x_{1}, \ldots, x_{D}, p_{1}, \ldots, p_{D}\right)}\right| d^{D} x d^{D} p
$$

For generality we consider here $D$-dimensional case. For the derivatives we have:

$$
\begin{gathered}
\frac{\partial x_{i}^{\prime}}{\partial x_{j}}=\delta_{i j}+\frac{\partial \dot{x}_{i}}{\partial x_{j}} \delta t ; \quad \frac{\partial x_{i}^{\prime}}{\partial p_{j}}=\frac{\partial \dot{x}_{i}}{\partial p_{j}} \delta t \\
\frac{\partial p_{i}^{\prime}}{\partial x_{j}}=\frac{\partial \dot{p}_{i}}{\partial x_{j}} \delta t ; \quad \frac{\partial p_{i}^{\prime}}{\partial p_{j}}=\delta_{i j}+\frac{\partial \dot{p}_{i}}{\partial p_{j}} \delta t .
\end{gathered}
$$


We calculate the Jacobian in the relation (43) up to the first order over the infinitesimal time translation $\delta t$. So under this approximation the Jacobian can be written in the form:

$$
J=\left|\frac{\partial\left(x_{1}^{\prime}, \ldots, x_{D}^{\prime}, p_{1}^{\prime}, \ldots, p_{D}^{\prime}\right)}{\partial\left(x_{1}, \ldots, x_{D}, p_{1}, \ldots, p_{D}\right)}\right|=1+\left(\frac{\partial \dot{x}_{i}}{\partial x_{i}}+\frac{\partial \dot{p}_{i}}{\partial p_{i}}\right) \delta t
$$

So we calculate:

$$
\begin{array}{r}
\frac{\partial \dot{x}_{i}}{\partial x_{i}}+\frac{\partial \dot{p}_{i}}{\partial p_{i}}=\frac{\partial}{\partial x_{i}}\left(\left\{x_{i}, x_{j}\right\} \frac{\partial H}{\partial x_{j}}+\left\{x_{i}, p_{j}\right\} \frac{\partial H}{\partial p_{j}}\right)+\frac{\partial}{\partial p_{i}}\left(\left\{p_{i}, x_{j}\right\} \frac{\partial H}{\partial x_{j}}+\left\{p_{i}, p_{j}\right\} \frac{\partial H}{\partial p_{j}}\right) \\
=\frac{\partial}{\partial x_{i}}\left[\left\{x_{i}, x_{j}\right\}\right] \frac{\partial H}{\partial x_{j}}+\left\{x_{i}, x_{j}\right\} \frac{\partial^{2} H}{\partial x_{i} \partial x_{j}}+\frac{\partial}{\partial x_{i}}\left[\left\{x_{i}, p_{j}\right\}\right] \frac{\partial H}{\partial p_{j}}+\left\{x_{i}, p_{j}\right\} \frac{\partial^{2} H}{\partial x_{i} \partial p_{j}}+ \\
\frac{\partial}{\partial p_{i}}\left[\left\{p_{i}, x_{j}\right\}\right] \frac{\partial H}{\partial x_{j}}+\left\{p_{i}, x_{j}\right\} \frac{\partial^{2} H}{\partial p_{i} \partial x_{j}}+\frac{\partial}{\partial p_{i}}\left[\left\{p_{i}, p_{j}\right\}\right] \frac{\partial H}{\partial p_{j}}+\left\{p_{i}, p_{j}\right\} \frac{\partial^{2} H}{\partial p_{i} \partial p_{j}} \\
=2\left(\alpha x_{k} \frac{\partial H}{\partial p_{k}}-\beta p_{k} \frac{\partial H}{\partial x_{k}}+\sqrt{\alpha \beta}\left[p_{k} \frac{\partial H}{\partial p_{k}}-x_{k} \frac{\partial H}{\partial x_{k}}\right]\right)
\end{array}
$$

For the element of phase space volume we have:

$$
d^{D} x^{\prime} d^{D} p^{\prime}=d^{D} x d^{D} p\left[1+2\left(\alpha x_{k} \frac{\partial H}{\partial p_{k}}-\beta p_{k} \frac{\partial H}{\partial x_{k}}+\sqrt{\alpha \beta}\left[p_{k} \frac{\partial H}{\partial p_{k}}-x_{k} \frac{\partial H}{\partial x_{k}}\right]\right) \delta t\right]
$$

Let us consider:

$$
\begin{array}{r}
1+\alpha x^{\prime 2}+\beta p^{\prime 2}+2 \sqrt{\alpha \beta}\left(\mathbf{x}^{\prime}, \mathbf{p}^{\prime}\right)=1+\alpha\left(x_{i}+\delta x_{i}\right)^{2}+\beta\left(p_{i}+\delta p_{i}\right)^{2} \\
+2 \sqrt{\alpha \beta}\left(x_{i}+\delta x_{i}, p_{i}+\delta p_{i}\right) \simeq 1+\alpha x^{2}+\beta p^{2}+2 \sqrt{\alpha \beta}(\mathbf{x}, \mathbf{p}) \\
+2\left(\alpha\left(x_{i}, \dot{x}_{i}\right)+\beta\left(p_{i}, \dot{p}_{i}\right)+\sqrt{\alpha \beta}\left[\left(x_{i}, \dot{p}_{i}\right)+\left(p_{i}, \dot{x}_{i}\right)\right]\right) \delta t=\left(1+\alpha x^{2}+\beta p^{2}\right. \\
+2 \sqrt{\alpha \beta}(\mathbf{x}, \mathbf{p}))\left[1+2\left(\alpha x_{k} \frac{\partial H}{\partial p_{k}}-\beta p_{k} \frac{\partial H}{\partial x_{k}}+\sqrt{\alpha \beta}\left[p_{k} \frac{\partial H}{\partial p_{k}}-x_{k} \frac{\partial H}{\partial x_{k}}\right]\right) \delta t\right]
\end{array}
$$

Making use of relations (43) and (44) we conclude that the following weighted phase space volume is invariant under infinitesimal time translations:

$$
\frac{d^{D} x d^{D} p}{1+\alpha x^{2}+\beta p^{2}+2 \sqrt{\alpha \beta}(\mathbf{x}, \mathbf{p})}
$$

\section{References}

[1] N. Arkani-Hamed, S. Dimopoulos, and G. Dvali, Phys. Lett. B 429, 263 (1998);

[2] N. Arkani-Hamed, S. Dimopoulos, and G. Dvali, Phys. Rev. D. 59, 086004 (1999);

[3] I. Antoniadis,N. Arkani-Hamed, S. Dimopoulos, and G. Dvali, Phys. Lett. B 436, 257 (1998).

[4] L. Randall, R. Sundrum, Phys. Rev. Lett. 83, 3370 (1999); Phys. Rev. Lett. 83, 4690 (1999).

[5] S. B. Giddings and S. Thomas, Phys. Rev. D 65056010 (2002);

[6] D. M. Eardley and S. B. Giddings, Phys. Rev. D 66, 044011 (2002);

[7] H. Yoshino and Y. Nambu, Phys. Rev. D 66, 065004 (2002);

[8] H. Yoshino and Y. Nambu, Phys. Rev. D 67, 024009 (2003);

[9] E. Berti, M. Cavaglia and M. Gualtieri, Phys. Rev. D. 69, 124011 (2004); 
[10] M. Cavaglia, R. Godang, L. M. Cremaldi and D. J. Summers, JHEP 0706, 055, (2007);

[11] P. Kanti, Lect. Notes Phys.769, 387, (2009);

[12] M. B. Voloshin, Phys. Lett. B 524, 376 (2002); Erratum-ibid. B 605, 426 (2005);

[13] V. S. Rychkov, Phys. Rev. D 70, 044003 (2004).

[14] R. J. Adler, P. Chen and D. I. Santiago, Gen. Rel. Grav. 33, 2101 (2001).

[15] M. Cavaglia and S. Das, Class. Quant. Grav. 21, 4511 (2004).

[16] F. Scardigli, arXiv:0809.1832,

[17] P. Nicolini, Int. J. Mod. Phys. A 24, 1229, (2009).

[18] B. A. Powell and W. H. Kinney, Phys. Rev. D. 76, 063512 (2007);

[19] I-C. Wang and K.-W. Ng, Phys. Rev. D. 77, 083501 (2008).

[20] F. Scardigli, C. Gruber, P. Chen, Phys. Rev. D, 82, 063507 (2011).

[21] D. J. Gross and P. F. Mende, Nucl. Phys. B 303, 407 (1988).

[22] E. Witten, Phys. Today 49, 24 (1996).

[23] M. Maggiore, Phys. Lett. B 304, 65 (1993);

[24] F. Scardigli, Phys. Lett. B 452, 39, (1999).

[25] B. S. Kay, arXiv: hep-th/9802172;

[26] B.S. Kay, Class.Quant.Grav.15:L89-L98,1998;

[27] B. S. Kay and V. Abyaneh, arXiv: 0710.0992.

[28] C. Bambi and F.R. Urban, Class. Quant. Grav. 25, 095006, (2008).

[29] A. Kempf, J. Math. Phys. 35, 4483 (1994).

[30] R. Banerjee, K. Kumar and D. Roychowdhury, JHEP 1103:060, (2011);

[31] S. Mignemi, arXiv: 1111.0201;

[32] Y. S. Myung, Y.-W. Kim and Y.-J. Park, Phys. Lett. B, 393, 393, (2007).

[33] T. Padmanabhan, Mod. Phys. Lett. A 25, 1129, (2010).

[34] M.-I. Park, Phys. Lett. B 659, 698, (2008).

[35] B. Bolen, M. Cavaglia, Gen. Rel. Grav. 37, 1255, (2005).

[36] M. Visser, Int. J. Mod. Phys. 12, 649, (2003).

[37] F. R. Tangherlini, Nuovo Cim. 27, 636 (1963).

[38] R. C. Myers and M. J. Perry, Ann. Phys. 172, 304 (1986).

[39] S. W. Hawking and D. N. Page, Commun. Math. Phys. 87, 577 (1983);

[40] P. Majumdar, Class. Quant. Grav. 24, 1747, (2007).

[41] P. Nicolini, arXiv: 1202.2102.

[42] L. N. Chang, D. Minic, N. Okamura and T. Takeuchi, Phys. Rev. D 65, 125028, (2002).

[43] R. Casadio, B. Harms, Phys. Rev. D. 64, 024016, (2001);

[44] R. Casadio, B. Harms, Entropy 13, 502, (2011).

[45] T. V. Fityo, Phys. Lett. A 372, 5872, (2008). 